\title{
Involucramiento parental mediante el juego en colegio semi rural en Chile: contribuyendo al desarrollo de niñas, niños y adolescentes
}

(Parental involvement through game in semi rural school in Chile: contributing to the development of girls, boys and adolescents.)

\author{
Marcelo Alfredo Villalón Calderón \\ Universidad de Chile. Escuela de Salud Pública (Chile) \\ mavillal@uchile.cl \\ Paola Elena Gacett Gasetet \\ Universidad Católica Silva Henríquez (Chile) \\ pgacett@colegiodesantamaria.com \\ Juan Eduardo Salazar Acevedo \\ Universidad San Sebastián (Chile) \\ juane.salazara@gmail.com \\ Catalina Cecilia Poblete Achondo \\ Carrera de Psicología, Universidad Santo Tomás (Chile) \\ doctorapoblete@gmail.com
}

Fecha recepción:07/05/2021

Páginas 74-81

Fecha aceptación:25/05/2021

\section{Resumen.}

En el contexto de acompañar y favorecer un desarrollo integral entre niños, niñas y adolescentes (NNA) de un colegio rural de la comuna de El Monte, Chile. Dado el conocimiento existente que asocia un desarrollo más pleno y con menores posibilidades de toma de decisión con tendencia al riesgo en este grupo etario cuando existe un mayor involucramiento parental, un grupo interdisciplinario de profesionales está implementando el juego como una actividad que permite la articulación de objetivos pedagógicos de los ámbitos de formación genérica (actitudinal) y cognitiva a largo plazo mediante la participación activa de los adultos con quienes NNA mantienen un vínculo. Utilizando como marco de referencia la efectividad de la prevención ambiental, se revisan algunas nociones centrales. Entre ellas, la alfabetización emocional como factor protector de salud, la importancia de los límites que se adecúan en la interacción con los adultos según etapa del ciclo vital en NNA donde el juego puede actuar con un rol modelador, la interacción entre el neurodesarrollo y la exposición en este periodo a agentes externos (un ejemplo concreto es la exposición a drogas y alcohol que, retardada en su inicio, reduce la probabilidad de adicciones a futuro), y la importancia del rol de una comunidad de adultos con un mismo discurso, dispuestos a cuidar y generar condiciones para que lo anterior se plasme. Este es un 
primer reporte que busca ser un precedente para las investigaciones en terreno, que están en desarrollo.

Palabras clave: involucramiento parental; juegos; desarrollo infantil y adolescente

\begin{abstract}
.
In the context of accompanying and promoting fuller development among boys, girls and adolescents (NNA) in a rural school in the El Monte district, Chile. Given the existing knowledge that associates a fuller development and with fewer possibilities of decision-making with a tendency to risk in this age group when there is greater parental involvement, an interdisciplinary group of professionals is implementing the game as an activity that allows the articulation of pedagogical objectives of the areas of generic (attitudinal) and cognitive training in the long term through the active participation of adults with whom children and adolescents maintain a bond. Taking the effectiveness of environmental prevention as a frame of reference, some central notions are reviewed. Among them, emotional literacy as a protective factor of health, the importance of the limits that are adapted in the interaction with adults according to the stage of the life cycle in NNA where the game can act with a modeling role, the interaction between neurodevelopment and exposure in this period to external agents (a concrete example is exposure to drugs and alcohol that, delayed in their onset, reduces the probability of addictions in the future) and the importance of the role of a community of adults with the same discourse, willing to care and generating conditions for the above to be reflected. This is a first report that seeks to be a precedent for field investigations, which are under development.
\end{abstract}

Keywords: parental involvement; games; child and adolescent development

\title{
1.-Introducción.
}

El presente artículo es el primero de una serie acerca del diseño e implementación de un proceso cuyo propósito es enriquecer el desarrollo de niñas, niños y adolescentes en un espacio escolar en Chile. Reconociendo el involucramiento parental como una estrategia central para promover esto en las nuevas generaciones, el juego surge como una actividad que permite articular objetivos pedagógicos de los ámbitos de formación genérica (actitudinal) y cognitiva en el largo plazo mediante la participación activa de los adultos con quienes niñas, niños y adolescentes mantienen un vínculo.

\section{2.-Desarrollo.}

En nuestro quehacer, acompañando procesos para fortalecer el desarrollo y prevenir conductas de riesgo en Niños, Niñas y Adolescentes (NNA), hemos constatado que la interacción con un adulto significativo a través del juego contribuye a crear y/o fortalecer una relación vinculante sana, afectiva, de reconocimiento mutuo, que facilita alfabetización emocional favoreciendo un neurodesarrollo más pleno. 
En todo el período del ciclo vital parece importante rescatar algunos aportes que desde la neurociencia permiten enfocarnos en el por qué hoy día esta temática es relevante y por qué tenemos que como adultos conocer ciertas premisas para que logremos ser promotores de un desarrollo pleno de nuestros NNA. Se sabe que desde que estamos gestando hasta los 21 años, custodiar el proceso de neurodesarrollo disminuye las probabilidades de mermar habilidades que se están todavía configurando. Un ejemplo de esto son los estudios de la doctora Nora Volkow y su equipo que han evidenciado que retrasar la edad de inicio de consumo de sustancias (alcohol y otras drogas) permite evitar un cambio a nivel estructural de poda y/o mielinización de distintas áreas del cerebro, lo que está ligado directamente con disminuir la probabilidad de una adicción en la adultez (Volkow et al., 2016, p.374).

Entonces, a partir del neurodesarrollo, nos damos cuenta que el hecho de proteger un cerebro vulnerable, no sólo de las drogas sino también de eventos vitales estresantes, incluidos los asociados a pobreza (Encina, 2008, citado en Torres et al., 2018), lo que estamos haciendo es que el cerebro de nuestros NNA tengan su tiempo de resguardo para un desarrollo pleno de sus potencialidades. Si a esto le sumamos la interacción a través del juego con un adulto sano, la generación de ese espacio podría ser, según la edad del NNA, parte de esos factores protectores. En este sentido el juego se manifiesta como una manera de reconocerse y construir leguaje y vínculos significativos. Huizinga (1998) nos hace una aproximación desde la posibilidad de Recrear la realidad, permitiendo a sus participantes de "ausentarse" de su realidadrealidad, generando un nuevo espacio horizontal donde es posible establecer nuevos códigos de lenguaje, con sus propias leyes resignificando símbolos propios del contexto acordado.

En la etapa preescolar los estudios de la psicóloga chilena Neva Milicic (2017), en relación a la Alfabetización Emocional, que significa en lo concreto y en simple que el NN pueda ponerle nombre a sus emociones, las reconozca, sepa también, por ejemplo, por qué le pusieron el nombre que tiene o por qué le gusta comer porotos con riendas (plato típico chileno), todo lo cual podría verse facilitado (nuevamente) en la interacción mediante el juego. Que permitiría entregar relatos simples por el adulto de conocimientos narrados por él (padre, madre, tíos, abuelas) que acompañan a ese NN. Hoy se sabe que generar ese cúmulo de historias, narraciones le permitirán proyectar la posibilidad en la adolescencia de lograr una identidad e indirectamente ir hacia una toma de decisión que tienda al autocuidado. Relatos que resultan esenciales para que, también en la etapa escolar, se alcancen capacidades de resiliencia, pues los NNA han recogido elementos de cómo los adultos que les rodean han sido capaces de sortear momentos de adversidad y a la vez recuerdos de momentos agradables relacionados, por ejemplo, con la risa, generador de endorfinas, un buen protector neuronal. Las posibilidades que plantean algunos juegos, permiten conectar con estas historias, además de establecer vínculos de significantes comunes en el imaginario social y hacia la experiencia subjetiva, describiendo nuevamente los elementos e impactando el entendimiento de la lengua misma y por consiguiente la respuesta frente a la lectura previa (Randazzano, 2012). 
Pensemos en otro aspecto, la empatía, que es tan importante para luego en una etapa adulta plantearnos ante otro adulto, reconocer mi yo y el del otro y no terminar ejerciendo actos abusivos resultado de no considerar al otro como a uno mismo (Ábalos, 2020). Esa empatía que, si se desarrolla, brinda la capacidad de reconocer que existen "otros", ejercicio que el adulto modela también en esta interacción a través del juego.

Hemos dado algunas luces de que este proceso de ciclo vital es más pleno cuando hay adultos que acompañan a los NNA y un ejemplo de ese acompañar, es esta propuesta de "jugar". Este acompañamiento, que se conoce también como Parentalidad Positiva o Involucramiento Parental (Burkhart, 2019), se refiere a cómo yo en mi rol de adulto reconociendo a ese NNA como sujeto de derecho (con el derecho primario a ser cuidado, respetado, acompañado), como un legítimo otro y en pleno proceso de neurodesarrollo, (cuido). Pero no sólo un cuidado directo de un padre 0 madre biológica. Lo hago también como parte de una comunidad de adultos que compartimos el propósito de promover las mejores condiciones para el desarrollo de las nuevas generaciones.

Ese colectivo donde un NNA es considerado por cada adulto como su "propio hijo", al estilo africano "para educar a un NNA hace falta una tribu" (Anónimo, Proverbio africano), se configura, así como un espacio en que el juego puede potenciar. Esto es, teniendo un grupo de adultos implicados en el proceso de acompañamiento de NNA y escogiendo el juego específico con adecuación a la edad/periodo del ciclo vital, se propician condiciones para que a nivel familiar, escolar y social se articulen relaciones que plasmen valores propios de cada cultura, de vinculación significativa respecto del bien del otro a nivel personal y comunitario, independientemente de nuestra diversidad (edad, nacionalidad u otra condición). Así, la estrategia del juego tiene la potencialidad de representar un camino que contribuya a procesos de integración de personas y comunidades diversas, en un Chile que entre los censos de 1992 y 2017 muestra un crecimiento de la población inmigrante.

Una de las mejores y más actuales demostraciones de la eficacia de la aproximación descrita respecto de los beneficios para los NNA y la comunidad entera, es el logro de Islandia en disminuir conductas de riesgo al tener una estrategia que supone aumentar el tiempo que los NNA pasan con sus familias y que los espacios de ocio sean estructurados, supervisados y acompañados por un adulto (Kristjansson, 2010).

Hoy día existe evidencia que ese rol del adulto mediando la situación de estrés de un NNA (actuando como buffer), ha podido determinar que situaciones vividas no se conviertan en estrés tóxico, y por ende, protege de los daños que éste implica en las distintas dimensiones de la salud a lo largo de toda la vida.

Dado que los juegos tienen reglas con el fin explicitar un marco compartido, los visualizamos como espacios formativos sanos. El sentido de los límites, imprescindibles en el desarrollo de NNA se pueden distinguir de este modo de la noción habitual y reduccionista asociada al castigo (como a veces en nuestra cultura 
latinoamericana se interpreta) y manifestarse con una connotación favorable de cuidado (Burkhart, 2011). Los juegos se ofrecen como instancias para ilustrar cómo ejercer estos límites de manera adecuada, según cada fase del ciclo vital. En un preescolar y escolar, estos límites son puestos desde el adulto. Además de ser un espacio seguro donde el equivocarse es parte del proceso de aprendizaje y no conlleva consecuencias permanentes, es más, se plantea como necesario en la búsqueda de explorar nuevas estrategias que surgen de las hipótesis planteadas y las experiencias vividas, sean éstas ganando o perdiendo el juego (Plass, 2015).

¿Con qué fin? Se ha demostrado por ejemplo que los límites a esta edad disminuyen las inseguridades y los síntomas ansiosos, entre otros beneficios. Ahora bien, en la etapa de adolescencia, esa forma de poner límites cambia. Se debe ajustar y aparece la importancia de "acordar" entre el adolescente y su adulto responsable. ¿Por qué es tan importante este cambio de implementación, de mirada respecto a la postura de límites? En esta etapa del desarrollo (adolescencia), el límite no sólo sirve para segurizarle, sino para promover el "valor de la palabra" en el adolescente (ejercicio indirecto de reconocimiento del otro). Al estar "acordado" con los demás jugadores (origen etimológico, poner el corazón en común), el adolescente no sólo responde a ese yo externo (la figura del adulto con quien juega), que le pone límite, sino a su propio yo interno pues ha puesto de él para llegar a acordar ese límite y entonces al transgredirlo (esperable en esta etapa), también tiene que nuevamente restablecer ese vínculo con el adulto y reelaborar el valor de su propia palabra para seguir funcionando en una dinámica sana que le permite seguir disfrutando del juego.

\section{5.-Reflexiones finales.}

A modo de recomendación general y sintética, como nos sugiere y enseña Diego Salazar (2014), en un desarrollo sano de un NNA se requieren pocos NO, pero muy claros y precisos; aparejados de muchos SI, donde el adolescente se expresa logra vincularse y encuentra pares, ejercicio básico para su desarrollo integral. Entre estos sí se cuentan también los juegos, que se pueden transformar en intereses o hobbies, tan necesarios durante la etapa preescolar y escolar para que luego durante la adolescencia se enriquezcan y afinen los Sí que ya comenzaron a implementar en las etapas previas.

Sean los juegos empleados de manera aislada (sólo con la familia en las vacaciones) o sistemática (como parte de un programa en un establecimiento escolar que es la apuesta que estamos implementado en el espacio escolar en la ciudad de El Monte, Chile), se trata de recursos valiosos y a nuestro parecer subutilizados hasta ahora. Su uso ha demostrado tener beneficios múltiples en el desarrollo de NNA, que se pueden ver potenciados si se articulan con adultos involucrados que acompañan de manera creativa el futuro.

\section{6.-Referencias Bibliográficas}


Ábalos, M., P. (2020). Vergüenza. Abusos en la Iglesia Católica. Ediciones Universidad Alberto Hurtado.

Bedregal, P., Hernández, V., Prado, P., Castañón, C., Mingo, V., \& de la Cruz, R. (2010). Hacia la evaluación de "Chile Crece Contigo": Resultados psicosociales del estudio piloto. Revista Médica de Chile, 138(6), 791-793. https://doi.org/10.4067/S0034-98872010000600022

Bucci, M., Marques, S., Oh, D., Harris, N. (2016). Toxic Streets in Children and Adolescents. Adv. Pediatr. 63(1) 403 - 428. http://doi.org/10.1016/i.yapd.2016.04.002

Burkhart, G., Helmer S. (2019). Technical Report. Drug Prevention: exploring a system perspective. European monitoring Centre for Drugs and Drug Addiction. https://doi.org/10.2810/51693

Burkhart, G. Enviromental drug prevention in the EU (2011). Prevención ambiental de drogas en la Unión Europea. ¿Por qué es tan impopular este tipo de prevención? Why is it so impopular?. Adicciones, 2011. European monitoring Centre for Drugs and Drug Addiction. 23 (2): 399-44. https://doi.org/10.20882/adicciones.151

Branco, M., Linhares, M. (2016). Toxic Stress in Children and Adolescents. Adv Pediatr. 63(1) 403- 428. https://doi.org/10.1590/1982-02752018000100009

Canal TEDMED. (27 Enero 2015). Why do our brains get addicted? [Archivo de video] https://www.youtube.com/watch?v=Mnd2-al4LCU Acceso 26 marzo 2021.

Encina, J. (2008, 09 de mayo). Chile Crece Contigo: el efecto de la implementación de salas cunas públicas en participación laboral femenina, pobreza y distribución de

ingresos.

https://www.cl.undp.org/content/chile/es/home/library/poverty/documentos de trabajo/chile-crece-contigo--el-efecto-de-la-implementacion-de-salascun.html

Erickson, E. (2011). El ciclo vital completado I (2ª ed.). Paidós.

Erikson, E. (1968). Identity: Youth and Crisis. W.W. Norton \& Company.

Erikson, E. (2004). Sociedad y adolescencia (19 ed.). Siglo XXI

Fundación de Desarrollo Educativo (2017). Seis usos para una ludoteca en contexto escolar.8-10. https://www.observatoriodeljuego.cl/recursoeducativos/

Gilbert, L., Breiding, M. Merrick, M., Thompson, W., Ford, D., Dhingra, S. et al. (2015) Childhood adversity and adult chronic disease: An update from ten states and 
the district of Columbia. Am J Prev Med. 48(3) 345 - 349. http://doi.org/10.1016/i.amepre.2014.09.006

Jhonson, S., Riley, A., Granger, D., Riis, J. (2013). The science of early life Toxic stress for pediatric practice and advocacy. Pediatrics, 131(2) 319-327. https://doi.org/10.1542/peds.2012-0469

Koob, G.F., \& Volkow, N. D. (2016). Neurobiology of addiction: a neurocicuitry análisis. The Lancet Psychiatry, 3(8), 760- 773. https://doi.org/10.1016/s2215$\underline{0366(16) 00104-8}$

Kristjansson, A., James, J., Allegrante, J., Sigfusdottir, I, Helgason, A. (2010). Adolescent substance use, parental monitoring, and leisure-time activities: 12year outcomes of primary prevention in Iceland. Preventive Medicine, 51(2): 168-171.https://doi.org/10.1016/i.ypmed.2010.05.001 https://www.sciencedirect.com/science/article/abs/pii/S0091743510001763

Kristjansson, A., Mann, M., Thorisdottir, I, Allegrante J. Sifusdottir, I. (2020). Implementing the Icelandic Model for Preventing Adolesent Substance Use. Health Promot Pract, 21 (1): 70- 79. http://doi.org/10.1177/1524839919849033

Kristjansson, A., Mann, M., Sigfusson, J., Thorisdottir, I., Allegrante, J., Sigfusdottir, I., (2020) Development and Guiding Principles of the Icelandic Model for Preventing Adolescent Substance Use. Health Promot Pract, 21(1): 62- 69. http://doi.org/10.1177/1524839919849032

Milicic, N. (2017). Educando a los Hijos con Inteligencia Emocional. Aguilar.

National Scientific Council on the Developing Child. (2014). Excessive Stress Disrupts the Architecture of the Developing Brain: Working Paper 3. Center on the Developing Child, Harvard University. 1-12. https://developingchild.harvard.edu/wpcontent/uploads/2005/05/Stress Disrupts Architecture Developing Brain1.pdf Acceso 26 de Marzo 2021.

Plass, J., Homer, B., Kinzer, C. (2015). Foundations of Game Based Learning. RoutLedge, Taylor \& Francis Group, 50(4) 258-283. http://doi.org/10.1080/00461520.2015.1122533

Randazzano, F. (2012). Los imaginarios sociales como herramienta. Imagonautas 2(2) 77-96.

https://www.academia.edu/13838164/Los imaginarios sociales como herra $\underline{\text { mienta }}$ 
Shonkoff, J., Boyce, W., Mc Ewen, B. Neuroscience, Molecular Biology and the Childhood Roots of Health Disparities. J Am Med Assoc, 301 (21) 2252- 2259 http://doi.org/10.1001/jama.2009.754

Siegel, B., Dobbins, Earls, M., Garner, A., Pascoe, J., Wood, D. et al. (2012). Early childhood adversity, toxic stress and the role of the pediatrician: Translating developmental science into lifelong health. Pediatrics, 129(1) https://doi.org/10.1542/peds.2011-2662

Shirtcliff, E., Coe, C., Pollack, S. (2009). Early childhoos stress is associated with elevated antibody levels of herpes simplex virus type 1. Proc Natl Acad Sci USA, 106(8) 2963-29567. http://doi.org/10.1073/pnas.0806660106

Torres, A. Lopez Boo, F., Parra, V., Vazquez, C., Segura-Pérez, S., Cetin, Z., \& PérezEscamilla, R. (2018). Chile Crece Contigo: Implementation, results, and scalingup lessons. Chils: care, health and development, 44(1), 4-11. https://doi.org/10.1111/cch.12519

Volkow, N. D., Koob, G. F. \& McLellan, A. T. (2016). Neurobiologic advances from the brain disease model of addiction. New England Journal of Medicine, 374 (4), 363 - 371. https://doi.org/10.1056/NEJMra1511480

Volkow, N., \& Li, T. K. (2005). The neuroscience of addiction. Nature neurosciencie, 8(11). https://doi.org/10.1038/nrn1539

Volkow, N. D., Michaelides, M., \& Baler, R. (2019). The neuroscience of drug reward and addiction. Psychological reviews, 99(4), 2115-2140. https://doi.org/10.1152/physrev.00014.2018

Volkow, N.D., Gordon, J.A., Koob, G. F., Birnbaum, L. S., Clayton, J. A., Koroshetz, W. J., ... \& Croyle, R. T. (2020). An examination of Child and Adolescent Neurodevelopment Trough National Institutes of Health Studies. Public Health Reports, 135(2), 169-172. https://doi.org/10.1177/0033354919900889 